\title{
On Stateless Automata and P Systems *
}

\author{
Linmin Yang ${ }^{1}$, Zhe Dang ${ }^{1}$, and Oscar H. Ibarra ${ }^{2}$ \\ ${ }^{1}$ School of Electrical Engineering and Computer Science \\ Washington State University \\ Pullman, WA 99164, USA \\ lyang1@eecs.wsu.edu, zdang@eecs.wsu.edu \\ ${ }^{2}$ Department of Computer Science \\ University of California \\ Santa Barbara, CA 93106, USA \\ ibarra@cs.ucsb.edu
}

\begin{abstract}
We introduce the notion of stateless multihead two-way (respectively, oneway) NFAs and stateless multicounter systems and relate them to $\mathrm{P}$ systems and vector addition systems. In particular, we investigate the decidability of the emptiness and reachability problems for these stateless automata and show that the results are applicable to similar questions concerning certain variants of $\mathrm{P}$ systems, namely, token systems and sequential tissue-like $\mathrm{P}$ systems.
\end{abstract}

\section{Introduction}

There has been a flurry of research activities in the area of membrane computing (a branch of molecular computing) initiated seven years ago by Gheorghe Paun [8]. Membrane computing identifies an unconventional computing model, namely a $\mathrm{P}$ system, from natural phenomena of cell evolutions and chemical reactions. It abstracts from the way living cells process chemical compounds in their compartmental structures. Thus, regions defined by a membrane structure contain objects that evolve according to given rules. The objects can be described by symbols or by strings of symbols, in such a way that multisets of objects are placed in regions of the membrane structure. The membranes themselves are organized as a Venn diagram or a tree structure where one membrane may contain other membranes. By using the rules in a nondeterministic and maximally parallel manner, transitions between the system configurations can be obtained. A sequence of transitions shows how the system is evolving. At a high-level, a $\mathrm{P}$ system has the following key features:

*The work of Zhe Dang and Linmin Yang was supported in part by NSF Grant CCF-0430531. The work of Oscar H. Ibarra was supported in part by NSF Grants CCF-0430945 and CCF-0524136, and a Nokia Visiting Fellow scholarship at the University of Turku. 
- Objects are typed but addressless (i.e., without individual identifiers),

- Objects can transfer between membranes,

- Membranes themselves form a structure (such as a tree),

- Object transferring rules are in (either maximally or locally) parallel, and

- The system is stateless.

Biologically inspired computing models like $\mathrm{P}$ systems [9] are often stateless. This is because it is difficult and even unrealistic to maintain a global state for a massively parallel group of objects. Naturally, a membrane in a $\mathrm{P}$ system, which is a multiset of objects drawn from a given finite type set $\left\{a_{1}, \ldots, a_{k}\right\}$, can be modeled as having counters $x_{1}, \ldots, x_{k}$ to represent the multiplicities of objects of types $a_{1}, \ldots, a_{k}$, respectively. In this way, a $\mathrm{P}$ system can be characterized as a counter machine in a nontraditional form; e.g., without states, and with parallel counter increments/decrements, etc. The most common form of stateless counter machines are probably the Vector Addition Systems (VASs), which are well-studied. Indeed, VASs have been shown intimately related to certain classes of $\mathrm{P}$ systems [5]. However, with new applications of $\mathrm{P}$ systems in mind [10], the investigation of other classes of stateless automata deserve further investigation.

In this paper, we present some results in this direction, with applications to reachability problems for variants of $\mathrm{P}$ systems, namely, token systems and sequential tissue-like $\mathrm{P}$ systems.

\section{Preliminaries}

A nondeterministic multicounter automaton is a nondeterministic automaton with a finite number of states, a one-way input tape, and a finite number of integer counters. Each counter can be incremented by 1, decremented by 1, or stay unchanged. Besides, a counter can be tested against 0. It is well-known that counter machines with two counters have an undecidable halting problem. Thus, to study decidable cases, we have to restrict the behaviors of the counters. One such restriction is to limit the number of reversals a counter can make. A counter is n-reversal-bounded if it changes mode between nondecreasing and nonincreasing at most $n$ times. For instance, the following sequence of counter values:

$$
0,0,1,1,2,2,3,3,4,4,3,2,1,1,1,1, \ldots
$$

demonstrates only one counter reversal. A counter is reversal-bounded if it is $n$ reversal-bounded for some fixed number $n$ independent of computations. A reversalbounded nondeterministic multicounter automaton is a nondeterministic multicounter automaton in which each counter is reversal-bounded.

Let $Y$ be a finite set of variables over integers. For all integers $a_{y}$, with $y \in Y, b$ and $c$ (with $b>0$ ), $\sum_{y \in Y} a_{y} y<c$ is an atomic linear relation on $Y$ and $\sum_{y \in Y} a_{y} y \equiv_{b}$ $c$ is a linear congruence on $Y$. A linear relation on $Y$ is a Boolean combination (using $\neg$ and $\wedge$ ) of atomic linear relations on $Y$. A Presburger formula on $Y$ is the 
Boolean combination of atomic linear relations on $Y$ and linear congruences on $Y$. A set $P$ of tuples of nonnegative integers is Presburger-definable or a Presburger relation if there exists a Presburger formula $\mathcal{F}$ on $Y$ such that $P$ is exactly the set of the solutions for $Y$ that make $\mathcal{F}$ true. It is well known that Presburger formulas are closed under quantification.

Let $\mathrm{N}$ be the set of nonnegative integers and $n$ be a positive integer. A subset $S$ of $\mathrm{N}^{n}$ is a linear set if there exist vectors $v_{0}, v_{1}, \ldots, v_{t}$ in $\mathrm{N}^{n}$ such that $S=\{v \mid$ $\left.v=v_{0}+a_{1} v_{1}+\ldots+a_{t} v_{t}, \forall 1 \leq i \leq t, a_{i} \in \mathrm{N}\right\} . S$ is a semilinear set if it is a finite union of linear sets. It is known that $S$ is a semilinear set if and only if $S$ is Presburger-definable [2].

Let $\Sigma$ be an alphabet consisting of $n$ symbols $a_{1}, \ldots, a_{n}$. For each string (word) $w$ in $\Sigma^{*}$, we define the Parikh map of $w$, denoted by $p(w)$, as follows:

$$
p(w)=\left(i_{1}, \ldots, i_{n}\right) \text {, where } i_{j} \text { is the number of occurrences of } a_{j} \text { in } w \text {. }
$$

If $L$ is a subset of $\Sigma^{*}$, the Parikh map of $L$ is defined by $p(L)=\{p(w) \mid w \in L\}$. L is a semilinear language if its Parikh map $p(L)$ is a semilinear set.

The following result is known [4]:

Theorem $1 p(L(M))$ is an effectively computable semilinear set when $M$ is a reversal-bounded nondeterministic multicounter automaton.

Consider a reversal-bounded nondeterministic multicounter machine (which is a reversal-bounded nondeterministic multicounter automaton without input). Let $\left(j, v_{1}, \ldots, v_{k}\right)$ denote the configuration of $M$ when it is in state $j$ and counter $i$ has value $v_{i}$ for $1 \leq i \leq k$. Define $R(M)=\{(\alpha, \beta) \mid$ configuration $\alpha$ can reach configuration $\beta$ in 0 or more moves $\}$, which is called the reachability relation of $M$. Using Theorem 1, one can easily show that $R(M)$ is Presburger definable.

Theorem 2 The reachability relation of a reversal-bounded nondeterministic multicounter machine is Presburger definable.

An $n$-dimensional vector addition system (VAS) is specified by $W$, a finite set of vectors in $\mathbb{Z}^{n}$, where $\mathbb{Z}$ is the set of all integers (positive, negative, zero). For two vectors $x$ and $z$ in $\mathbb{N}^{n}$, we say that $x$ can reach $z$ if for some $j, z=x+v_{1}+\ldots+$ $v_{j}$, where, for all $1 \leq i \leq j$, each $v_{i} \in W$ and $x+v_{1}+\ldots+v_{i} \geq 0$. The Presburger reachability problem for VAS is to decide, given two Presburger formulas $P$ and $Q$, whether there are $x$ satisfying $P$ and $z$ satisfying $Q$ such that $x$ can reach $z$. The following theorem follows from the decidability of the reachability problem for VASs (which are equivalent to Petri nets) [7].

Theorem 3 The Presburger reachability problem for VAS is decidable.

\section{Stateless Multihead Two-way (One-way) NFAs/DFAs and Token Systems}

Let $\Sigma$ and $\Pi$ be two alphabets. An object of some type in $\Sigma$ (resp., $\Pi$ ) is called a standard object (resp., a token). Consider a chain (with length $n$ ) of membranes 
(i.e., membranes are organized as a linear structure)

$$
A_{1}, \ldots, A_{n}
$$

for some $n$. The chain is called initial if the following conditions are met:

1 Each $A_{i}$ holds exactly one standard object,

$2 A_{1}$ contains one token of each type in $\Pi$; the rest of the $A_{i}$ 's do not contain any tokens,

3 The standard object on the first membrane $A_{1}$ is of type $\triangleright \in \Sigma$ and the standard object on the last membrane $A_{n}$ is of type $\triangleleft \in \Sigma$; the membranes in between $A_{1}$ and $A_{n}$ do not contain any $\triangleright$-objects and $\triangleleft$-objects.

Let $\Pi^{\prime} \subseteq \Pi$ be given. The chain is called halting if we change the condition 2 in above into " $A_{n}$ contains one token of each type in $\Pi^{\prime}$." A rule specifies how objects are transferred between two neighboring membranes (i.e., $A_{i}$ and $A_{i+1}$ for $1 \leq i \leq n-1$ ) and is in one of the following two forms:

- $(a, p) \rightarrow$

- $(a, p)^{\leftarrow}$,

where $a \in \Sigma$ and $p \in \Pi$. For instance, when $(a, p) \rightarrow$ is applied on $A_{i}$, the $A_{i}$ must contain a standard $a$-object and a $p$-token. The result is to move the token from $A_{i}$ to $A_{i+1}$ (where $1 \leq i \leq n-1$ ). The semantics of $(a, p)^{\leftarrow}$ is defined similarly but the token $p$ moves from $A_{i+1}$ back to $A_{i}$. We are given a set of rules $R$ which are applied sequentially; i.e., each time, a rule and an $i$ are nondeterministically picked and the rule is applied on $A_{i}$. We are interested in studying the computing power of such token systems. Specifically, we focus on decision algorithms solving the following reachability problem: whether there is an $n$ and an initial chain with length $n$ such that, after a certain number of applications of rules in $R$, the initial chain evolves into a halting chain. Notice that an instance of a chain in the form of (1) is a special instance of tissue-like $\mathrm{P}$ systems [6] and in the future we will further study more general intra-membrane structures (such as graphs) than linear structures in (1). Also note that, in the reachability problem, the chain is not given. Instead, we are looking for a desired chain. This is different from the case for a tissue-like $\mathrm{P}$ system where a concrete instance (with the $n$ in (1) given) is part of the system definition.

One can generalize the aforementioned token systems by allowing some of the rules synchronized; i.e., a synchronized rule in the form of

$$
\left[r_{1}, r_{2}, \ldots, r_{k}\right]
$$

for some $k$ and distinct rules $r_{1}, \ldots, r_{k}$. The semantics of the synchronized rule is to apply each $r_{i}$ at the same time. For instance, a synchronized rule $\left[(a, p) \rightarrow,(b, q)^{\leftarrow}\right]$, when applied, is to nondeterministically pick an $A_{i}$ and $A_{j}$ (where $i$ could be the same as $j$ ) and apply the rule $(a, p) \rightarrow$ on $A_{i}$ and the rule $(b, q)^{\leftarrow}$ on $A_{j}$ (whenever both are applicable). Such systems with synchronized rules are called generalized 
token systems and we can raise the same reachability problem for generalized token systems.

We first observe that the (generalized) token systems are essentially the same as stateless multihead two-way NFAs $M$ studied in the following, where each input tape cell corresponds to a membrane in (1) and each token corresponds to a two-way head. The stateless NFA $M$ is equipped with an input on alphabet $\Sigma$ and heads $H_{1}, \ldots, H_{k}$ for some $k$. The heads are two-way, the input is read-only, and there are no states. An $H_{i}$-move (also called a local move) $\mathrm{MOVE}_{i}$ of the NFA can be described as a triple $\left(H_{i}, a, D\right)$, where $H_{i}$ is the head involved in the move, $a$ is the input symbol under the head $H_{i}$, and $D \in\{+1,-1,0\}$ meaning that, as a result of the move, the head $H_{i}$ goes to the right, goes to the left, or simply stays. When a head $H_{i}$ tries to execute a local move $\left(H_{i}, a, D\right)$, it requires that the symbol under $H_{i}$ must be $a$, otherwise $M$ just crashes. A generalized move is in the form of $\left(H_{i}, \mathcal{S}, \mathcal{D}\right)$, where $\mathcal{S}$ is a set of symbols, and $\mathcal{D}$ is a set of directions (i.e., $+1,-1,0$ ). When executing a generalized move $\left(H_{i}, \mathcal{S}, \mathcal{D}\right)$, the symbol $H_{i}$ reads must belong to $\mathcal{S}$, and $H_{i}$ nondeterministically picks a direction from $\mathcal{D}$.

Note that a local move is a special case of a generalized move. An instruction of $M$ is a sequence of local or general moves, in the form of [MOVE $i_{i_{1}}, \mathrm{MOVE}_{i_{2}}$, $\left.\ldots, \mathrm{MOVE}_{i_{m}}\right]$, for some $m, 1 \leq m \leq k$, and $i_{1}<\ldots<i_{m}$. (If $m=1$, the instruction is simply called a local instruction.) When the instruction is executed, the heads $H_{i_{1}}, \ldots, H_{i_{m}}$ perform the moves $\mathrm{MOVE}_{i_{1}}, \ldots, \mathrm{MOVE}_{i_{m}}$, respectively and simultaneously. Any head falling off the tape will cause $M$ to crash. The NFA $M$ has a finite set of such instructions. At each step, it nondeterministically picks a maximally parallel set of instructions to execute. $M$ has a set of accepting heads $F \subseteq\left\{H_{1}, \ldots, H_{k}\right\}$. For most constructions in this paper, $F$ consists of all the heads. Initially, all heads are at the leftmost cell of the input tape. $M$ halts and accepts the input when the accepting heads are all at the rightmost cell. We assume that the input tape of $M$ has a left end marker $\triangleright$ and a right end marker $\triangleleft$. Thus, for any input $a_{1} \ldots a_{n}, n \geq 2, a_{1}=\triangleright, a_{n}=\triangleleft$, and for $2 \leq i \leq n-1$, each $a_{i}$ is different from the end markers.

We emphasize that in a stateless multihead one-way (two-way) NFA, at each step, the application of the instructions is "maximally parallel", i.e., all instructions that can be applied to the heads must be applied. Note that, in general, the set of instructions that can be applied maximally parallel need not be unique. If at most $m$ instructions are applicable at each step, then we say the machine is $m$-maxpar.

A stateless one-way (two-way) DFA is one in which at each step of the computation, at most one maximally parallel set of instructions is applicable.

Our first result is the following:

Theorem 4 The reachability problem for generalized token systems is undecidable. The problem is decidable for token systems.

The first part of Theorem 4 is a direct consequence of the next theorem. The second part follows from the fact that the emptiness problem for two-way NFAs is decidable.

Theorem 5 The emptiness problem for stateless (1-maxpar) 3-head one-way DFAs is undecidable. 
Proof. Given a deterministic TM $Z$, let $A_{Z}=C_{1} \# C_{2} \# \ldots \# C_{n}$ be the halting computation of $Z$ starting on blank tape. Hence $C_{1}$ is the initial configuration (on blank tape), $C_{n}$ is the halting configuration, and $C_{i+1}$ is the direct successor of $C_{i}$. We assume that $n \geq 2$. Let $\Gamma$ and $Q_{Z}$ be the tape alphabet and state set, respectively, of $Z$.

Clearly, from $Z$, we can construct a 2-head one-way DFA $M_{0}$ (with states) with heads $H_{1}$ and $H_{2}$ and input alphabet $\Sigma=\Gamma \cup Q_{Z} \cup\{\#\}$, which accepts a nonempty language (actually only the string $A_{Z}$ ) iff $Z$ halts. Because from configuration $C_{i}$ the next step of $Z$ that results in configuration $C_{i+1}$ may move its read-write head left, $H_{2}$ may not always move to the right at every step in $M_{0}$ 's computation. However, we can modify $M_{0}$ into another two-head one-way DFA $M$ by putting "dummy" symbols $\alpha$ 's on the tape so that $H_{2}$ can read these symbols instead of not moving right. $H_{1}$, of course, ignores these dummy symbols. $M$ has now the property that $H_{2}$ always moves to the right at every step in the computation until $M$ accepts. Clearly, $L\left(M_{0}\right)=\emptyset$ if and only if $L(M)=\emptyset$, and if and only if $Z$ does not halt on blank tape. We may assume that $M$ accepts with $H_{2}$ falling off the right end of the tape in a unique accepting state $f$. (This assumption on $\mathrm{H}_{2}$ falling off the right end of the tape should not be confused with the condition that in a stateless automaton, a head falling off the tape will cause the machine to crash.) We also assume that there are no transitions from state $f$. Let $Q_{M}$ be the state set of $M$.

Thus, if $\delta\left(q, a_{1}, a_{2}\right)=\left(p, d_{1}, d_{2}\right)$, then $d_{2}=+1$. This transition is applicable if $M$ is currently in the state $q$ and the heads $H_{1}$ and $H_{2}$ are reading $a_{1}$ and $a_{2}$, respectively. When the transition is applied, $H_{2}$ moves right, $H_{1}$ moves right or remains stationary depending on whether $d_{1}$ is +1 or 0 , and $M$ enters state $p$.

We construct a stateless 3-head one-way DFA $M^{\prime}$ to simulate $M$. The heads of $M^{\prime}$ are $H_{1}, H_{2}$, and $H_{3}$. The input alphabet of $M^{\prime}$ is $\left(\Sigma \times Q_{M} \cup\{\triangleright, \triangleleft\}\right)$ ( $\triangleright$ and $\triangleleft$ are left and right end markers for the input to $M^{\prime}$.) The instructions of $M^{\prime}$ are as follows:

1. $\left[\left(H_{1}, \triangleright, 0\right),\left(H_{2}, \triangleright, 0\right),\left(H_{3}, \triangleright,+1\right)\right]$.

2. $\left[\left(H_{1}, \triangleright,+1\right),\left(H_{2}, \triangleright,+1\right),\left(H_{3},(a, q),+1\right)\right]$ for every $a \in \Sigma$ and every $q \in Q_{M}$.

3. Suppose $\delta\left(q, a_{1}, a_{2}\right)=\left(p, d_{1}, d_{2}\right)$ and $p \neq f$, then $\left[\left(H_{1},\left(a_{1}, s\right), d_{1}\right),\left(H_{2},\left(a_{2}, q\right),+1\right),\left(H_{3},(b, p),+1\right)\right]$ is a rule for every $s \in Q_{M}$ and every $b \in \Sigma$.

4. Suppose $\delta\left(q, a_{1}, a_{2}\right)=\left(f, d_{1},+1\right)$, then $\left[\left(H_{1},\left(a_{1}, s\right), d_{1}\right),\left(H_{2},\left(a_{2}, q\right),+1\right),\left(H_{3}, \triangleleft, 0\right)\right]$ is a rule for every $s \in Q_{M}$.

5. $\left[\left(H_{1},(a, q),+1\right),\left(H_{2},(b, p),+1\right),\left(H_{3}, \triangleleft, 0\right)\right]$ is a rule for every $a, b \in \Sigma$ and $q, p \in Q_{M}$.

6. $\left[\left(H_{1},(a, q),+1\right),\left(H_{2}, \triangleleft, 0\right),\left(H_{3}, \triangleleft, 0\right)\right]$ is a rule for every $a \in \Sigma$ and $q \in Q_{M}$.

$M^{\prime}$ accepts if and only if all three heads are on $\triangleleft$. From the construction, it is clear that $M^{\prime}$ is a stateless 3-head one-way DFA, and $L(M)=\emptyset$ if and only if $Z$ does not halt on blank tape. The result follows from the undecidability of the halting problem for TMs on blank tape. 
It is an interesting open question whether the 3 heads in the above theorem can be reduced to 2 , even if the 2 heads are allowed to be two-way. Note that in the theorem, all 3 heads are involved in every instruction. We can strengthen this result by a more intricate construction. Define a stateless $k$-head one-way 2-move NFA (DFA) to be one where in every instruction, at most two heads are involved. Then we have:

Theorem 6 The emptiness problem for stateless 3-head one-way 2-move DFAs is undecidable.

Proof. Let $M$ be the 2-head one-way DFA with states constructed in the previous proof. The transition $\delta(q, a, b)=\left(p, d_{1},+1\right)$ of $M$ can be represented by the tuple

$$
\left[q,\left(H_{1}, a, d_{1}\right),\left(H_{2}, b,+1\right), p\right]
$$

Suppose $M$ has $n$ such transitions, and we number them as $1, \ldots, n$. We may assume that $M$ starts its computation with rule number 1 .

Note that $H_{2}$ moves to the right at every step, and that $M$ accepts with $H_{2}$ falling off the right end of the tape in a unique accepting state $f$ and there are no transitions from state $f$.

We say that transition numbers $i$ and $j$ are compatible if they correspond to transition instructions $\left[q,\left(H_{1}, a, d_{1}\right),\left(H_{2}, b,+1\right), p\right]$ and $\left[p,\left(H_{1}, a^{\prime}, d_{1}^{\prime}\right),\left(H_{2}, b^{\prime},+1\right), r\right]$, respectively, for some states $q, p, r$, symbols $a, a^{\prime}, b, b^{\prime}$, and directions $d_{1}, d_{1}^{\prime}$.

The input alphabet of $M^{\prime}$ is $(\Sigma \times N \times \Delta) \cup\{\triangleright, \triangleleft\}$, where $N=\{1, \ldots, n\}$ (set of transition numbers of $M$ ) and $\Delta=\left\{\delta_{1}, \delta_{2}\right\}$ ( $\triangleright$ and $\triangleleft$ are the end markers of $M^{\prime}$ ). The heads of $M^{\prime}$ are $H_{1}, H_{2}, H_{3}$. The instructions of $M^{\prime}$ are defined as follows:

1. $\left[\left(H_{1}, \triangleright,+1\right)\right]$

2. $\left[\left(H_{3}, \triangleright, 0\right),\left(H_{2}, \triangleright,+1\right)\right]$

3. $\left[\left(H_{3}, \triangleright,+1\right),\left(H_{2},\left(c, 1, \delta_{1}\right), 0\right)\right]$ for every $c \in \Sigma$.

Suppose transition number $k$ corresponds to $\left[q,\left(H_{1}, a, d_{1}\right),\left(H_{2}, b,+1\right), p\right]$. Then the following instructions are in $M^{\prime}$ :

4. $\left[\left(H_{3},\left(b, k, \delta_{1}\right), 0\right),\left(H_{2},\left(b, k, \delta_{1}\right),+1\right]\right.$

5. $\left[\left(H_{3},\left(c, i, \delta_{1}\right),+1\right),\left(H_{2},\left(d, i, \delta_{2}\right), 0\right)\right]$, for every $1 \leq i \leq n$ and every $c, d \in \Sigma$.

6. $\left[\left(H_{1},(a, i, \delta), d_{1}\right),\left(H_{2},\left(c, k, \delta_{2}\right), 0\right)\right]$, for every $1 \leq i \leq n$, every $c \in \Sigma$, and every $\delta \in \Delta$.

7. $\left[\left(H_{3},\left(c, i, \delta_{2}\right), 0\right),\left(H_{2},\left(c, i, \delta_{2}\right),+1\right)\right]$, for every $1 \leq i \leq n$ and every $c \in \Sigma$.

8. $\left[\left(H_{3},\left(c, i, \delta_{2}\right),+1\right),\left(H_{2},\left(d, j, \delta_{1}\right), 0\right)\right]$, for every $1 \leq i, j \leq n$ with $i$ and $j$ compatible, and every $c, d \in \Sigma$.

9. $\left[\left(H_{3},\left(c, i, \delta_{2}\right),+1\right),\left(H_{2}, \triangleleft, 0\right)\right]$, every $c \in \Sigma$ and for every $1 \leq i \leq n$, with $i$ corresponding to a transition of the form $\left[q,\left(H_{1}, a^{\prime}, d_{1}\right),\left(H_{2}, b^{\prime},+1\right), f\right]$ for every state $q$ and $a^{\prime}, b^{\prime}$ in $\Sigma$. (Note that $f$ is the unique accepting state of $M$.) 
10. $\left[\left(H_{1},(c, i, \delta),+1\right),\left(H_{3}, \triangleleft, 0\right)\right]$, for every $1 \leq i \leq n$, every $c \in \Sigma$, and every $\delta \in \Delta$.

Define a homomorphism $h$ that maps each symbol $(\alpha, i, \delta)$ to $(i, \delta)$. Then we require that the homomorphic image of the input tape of $M^{\prime}$ (excluding the end markers) is a string in

$$
\left(1, \delta_{1}\right)\left(1, \delta_{2}\right)\left\{\left(1, \delta_{1}\right)\left(1, \delta_{2}\right), \ldots,\left(n, \delta_{1}\right)\left(n, \delta_{2}\right)\right\}^{*}
$$

so that a sequence of valid transitions can be executed properly. $H_{2}$ and $H_{3}$ are used for this purpose (i.e., to check the format).

$M^{\prime}$ accepts if and only if all heads are on the right end marker. From the construction, it is clear that $L\left(M^{\prime}\right)=\emptyset$ iff $L(M)=\emptyset$. The undecidability follows.

Next, we will study the emptiness problem when the inputs are restricted. Recall that a language is bounded if it is a subset of $a_{1}^{*} a_{2}^{*} \ldots a_{k}^{*}$ for some given symbols $a_{1}, \ldots, a_{k}$.

It is known [3] that if $M$ is a multihead one-way NFA with states but with bounded input, the language it accepts is a semilinear set effectively constructable from $M$. In fact, this result holds, even if $M$ has two-way heads, but the heads can only reverse directions from right to left or from left to right at most $r$ times, for some fixed $r$ independent of the input. It follows that Theorem 5 can not be strengthened to hold for one-way machines accepting bounded languages. However, for two-way machines, we can prove the following:

Theorem 7 The emptiness problem for stateless 5 -head two-way NFAs over bounded input is undecidable.

Proof. We show how a stateless 5-head two-way NFA $M^{\prime}$ can simulate a 2-counter machine $M$. Suppose $M$ has states $q_{1}, \ldots, q_{n}$, where we assume that $n \geq 3, q_{1}$ is the initial state, and $q_{n}$ is the unique halting state. Assume that both counters are zero upon halting, and the number of steps is odd. The transition of $M$ is of the form $\delta\left(q_{i}, s_{1}, s_{2}\right)=\left(q_{j}, d_{1}, d_{2}\right)$ where $s_{r}$ (sign of counter $\left.r\right)=0$ or + and $d_{r}$ (change in counter $r)=+1,0,-1$ for $r=1,2$.

A valid input to $M^{\prime}$ is a string of the form $\triangleright q_{1} q_{2} \ldots q_{n} a^{d} \triangleleft$ for some $d \geq 1$. We construct a stateless 5-head two-way NFA $M^{\prime}$ (with heads $H_{1}, H_{2}, H_{3}, C_{1}, C_{2}$ ) to simulate $M$. We begin with the following instructions:

$$
\begin{aligned}
& {\left[\left(H_{1}, \triangleright, 0\right),\left(H_{2}, \triangleright,+1\right)\right]} \\
& {\left[\left(H_{1}, \triangleright,+1\right),\left(H_{2}, q_{1},+1\right)\right]} \\
& {\left[\left(H_{1}, q_{1},+1\right),\left(H_{2}, q_{2},+1\right)\right]} \\
& \ldots \\
& \ldots \\
& {\left[\left(H_{1}, q_{n-1},+1\right),\left(H_{2}, q_{n},+1\right)\right]}
\end{aligned}
$$




$$
\begin{aligned}
& {\left[\left(H_{1}, q_{n},+1\right),\left(H_{2}, a,+1\right)\right]} \\
& {\left[\left(H_{1}, a,+1\right),\left(H_{2}, a,+1\right)\right]} \\
& {\left[\left(H_{1}, a,+1\right),\left(H_{2}, \triangleleft, 0\right)\right]}
\end{aligned}
$$

The instructions above check that the input is of the form $\triangleright q_{1} q_{2} \ldots q_{n} a^{d} \triangleleft$ for some $d \geq 1$. At the end of the process $H_{1}$ and $H_{2}$ are on the right end marker $\triangleleft$. Next add the following instructions:

$$
\begin{aligned}
& {\left[\left(H_{1}, \triangleleft,-1\right),\left(H_{2}, \triangleleft,-1\right),\left(H_{3}, \triangleright,+1\right)\right]} \\
& {\left[\left(H_{1}, t,-1\right),\left(H_{2}, t,-1\right),\left(H_{3}, q_{1}, 0\right)\right] \text { for all } t \neq q_{1}} \\
& {\left[\left(H_{1}, q_{1}, 0\right),\left(H_{2}, q_{1}, 0\right),\left(H_{3}, q_{1},+1\right)\right]} \\
& {\left[\left(H_{2}, q_{k},+1\right),\left(H_{3}, q_{2}, 0\right)\right] \text { for } 1 \leq k \leq n-1} \\
& {\left[\left(H_{2}, q_{k},-1\right),\left(H_{3}, q_{2}, 0\right)\right] \text { for } 2 \leq k \leq n} \\
& {\left[\left(H_{1}, q_{k},+1\right),\left(H_{3}, q_{3}, 0\right)\right] \text { for } 1 \leq k \leq n-1} \\
& {\left[\left(H_{1}, q_{k},-1\right),\left(H_{3}, q_{3}, 0\right)\right] \text { for } 2 \leq k \leq n}
\end{aligned}
$$

In the instructions above, if the symbol under $H_{3}$ is $q_{2}$ (resp., $q_{3}$ ), the machine positions $H_{2}$ (resp., $H_{1}$ ) to some nondeterministically chosen state (for use below).

Let $C_{1}$ and $C_{2}$ be the counters of $M$. Initially they are set to zero. In the instructions below, we use heads $C_{1}$ and $C_{2}$ to correspond to the counters.

If $\delta\left(q_{i}, s_{1}, s_{2}\right)=\left(q_{j}, d_{1}, d_{2}\right)$ where $s_{r}=0$ or + and $d_{r}=+1,0,-1$, then add the following instructions:

$$
\begin{aligned}
& {\left[\left(H_{1}, q_{i}, 0\right),\left(H_{2}, q_{j}, 0\right),\left(H_{3}, q_{2},+1\right),\left(C_{1}, t_{1}, d_{1}\right),\left(C_{2}, t_{2}, d_{2}\right)\right]} \\
& {\left[\left(H_{1}, q_{j}, 0\right),\left(H_{2}, q_{i}, 0\right),\left(H_{3}, q_{3},-1\right),\left(C_{1}, t_{1}, d_{1}\right),\left(C_{2}, t_{2}, d_{2}\right)\right]} \\
& \text { where } t_{r}=\triangleright \text { if } s_{r}=0 \text { and } t_{r} \neq \triangleright \text { if } s_{r}=+
\end{aligned}
$$

If $\delta\left(q_{i}, 0,0\right)=\left(q_{n}, 0,0\right)$ (i.e., the 2-counter machine $M$ halts in the unique state $q_{n}$ with both counters zero after an odd number of steps), then add the following instructions:

$$
\begin{aligned}
& {\left[\left(H_{1}, q_{i}, 0\right),\left(H_{2}, q_{n}, 0\right),\left(C_{1}, \triangleright,+1\right),\left(C_{2}, \triangleright,+1\right)\right]} \\
& {\left[\left(H_{1}, q_{i}, 0\right),\left(H_{2}, q_{n}, 0\right),\left(C_{1}, t,+1\right),\left(C_{2}, t,+1\right)\right] \text { for all } t \neq \triangleleft} \\
& {\left[\left(H_{r}, t,+1\right),\left(C_{1}, \triangleleft, 0\right),\left(C_{2}, \triangleleft, 0\right)\right] \text { for all } t \neq \triangleleft \text { and for } r=1,2,3}
\end{aligned}
$$

$M^{\prime}$ accepts if all heads $\left(H_{1}, H_{2}, H_{3}, C_{1}, C_{2}\right)$ are on the right end marker. It is easily verified that $M^{\prime}$ accepts the empty set if and only if $M$ does not halt.

The above theorem says that the emptiness problem is undecidable if the number of heads is 5 (i.e., fixed) but the size of the input alphabet is arbitrary. The next result shows that the emptiness problem is also undecidable if the size of input alphabet is fixed (in fact, can be unary) but the number of heads is arbitrary. 
Theorem 8 The emptiness problem for stateless multihead two-way NFAs is undecidable even when the input is unary but with the left end marker (resp., right end marker).

Proof. We only show the case with the left end marker. (The construction can easily be modified for the case with the right end marker.) We assume that the input has length at least 1, excluding the left end marker. We use a stateless NFA $M^{\prime}$ (whose input is unary with a left end marker) to simulate a two-counter machine $M$ with counters $C_{1}$ and $C_{2}$, and states $q_{0}, q_{1}, \ldots, q_{n}$. The idea is to use $\left\lceil\log _{2}(n+1)\right\rceil$ heads, $H_{1}, \ldots, H_{\left\lceil\log _{2}(n+1)\right\rceil}$, to control the states, and another two heads $H_{\left\lceil\log _{2}(n+1)\right\rceil+1}$ and $H_{\left\lceil\log _{2}(n+1)\right\rceil+2}$ to control the value of counters $C_{1}$ and $C_{2}$. The two-counter machine $M$ starts in state $q_{0}$, and $C_{1}=C_{2}=0$. Initially, all heads of $M^{\prime}$ are at the leftmost cell (i.e., $\triangleright$ ). If $H_{i}, 1 \leq i \leq\left\lceil\log _{2}(n+1)\right\rceil$, is at $\triangleright$, we consider it as 0 ; if $H_{i}$ is at the first $a$, we consider it as 1 . Hence $H_{\left\lceil\log _{2}(n+1)\right\rceil} \ldots H_{1}$ is a binary string (with $H_{\left\lceil\log _{2}(n+1)\right\rceil}$ as its most significant bit), which is used to encode the index of a state of $M$. Note that during the computation, $H_{i}, 1 \leq i \leq\left\lceil\log _{2}(n+1)\right\rceil$, only moves between the left end marker and the first $a$. We omit the details of the simulation of the instructions of $M$.

Theorems 7 and 8 are best possible, since we can not fixed both the number of heads and the size of the input alphabet and get undecidability. This is because for fixed $k$ and $n$, there are only a finite number of such stateless machines (also observed by Artiom Alhazov). Hence, the emptiness problem has a finite number of instances and therefore decidable.

A special case of Theorem 8 is when the input is unary and without end markers. In this case, the heads are initially at the leftmost input cell and the automaton accepts when the heads are all at the rightmost cell (we assume that there are at least two cells on the input). Using a VAS to simulate the multihead position changes, we have:

Theorem 9 The emptiness problem for stateless multihead two-way NFAs is decidable when the input is unary and without end markers.

Proof. Suppose we have a stateless NFA $M$ with $H_{1}, \ldots, H_{k}$ for some $k$, and with unary input

$$
a \ldots a,
$$

of length $B$ for some $B$. We can construct a corresponding VAS $G=\langle x, W\rangle$, where $x \in \mathbb{N}^{k}$ is the start vector, and $W$ is a finite set of vectors in $\mathbb{Z}^{k}$. Furthermore, we require that the maximal entry of any vector in $G$ can not exceed $B-1$; otherwise, $G$ crashes. In other words, $G$ is a bounded vector addition system. Since initially in $M$, all heads are at the leftmost cell, we set $x=(0, \ldots, 0)$ in $G$. If in $M$, there is an instruction $I=\left[\mathrm{MOVE}_{i_{1}}, \mathrm{MOVE}_{i_{2}}, \ldots, \mathrm{MOVE}_{i_{m}}\right]$, for some $m, 1 \leq m \leq k$, and $\operatorname{MOVE}_{i_{j}}=\left(H_{i_{j}}, a, D\right), 1 \leq j \leq m$, then in $G$ we have $v \in W$. For all $j, 1 \leq j \leq m$, the $i_{j}^{t h}$ entry of $v$ is 0 if $D=0$. The entry is 1 if $D=+1$. And, the entry is -1 if $D=-1$. All other entries are 0 .

Clearly, $M$ accepts a nonempty language iff, for some $B,(B-1, \ldots, B-1)$ is reachable in $G$; directly from Theorem 3 , the result follows. 


\section{Sequential Tissue-Like P Systems and Stateless Mul- ticounter Systems}

We now generalize the rules in a token system by allowing multiple objects to transfer from one membrane to another in (1); the result is a variant of a tissue-like $\mathrm{P}$ system [6] with sequential applications of rules [1]. More precisely, let $\Sigma=\left\{a_{1}, \ldots, a_{m}\right\}$. A sequential tissue-like $P$ system $G$ is a directed graph with $n$ nodes (for some $n$ ), where each node $i$ is equipped with a membrane $A_{i}$ which is a multiset of objects in $\Sigma$. In particular, we use $m$ counters $\vec{X}_{i}=\left(x_{i 1}, \ldots, x_{i m}\right)$ to denote the multiplicities of objects of types $a_{1}, \ldots, a_{m}$ in $A_{i}$, respectively. Each membrane $A_{i}$ is also associated with a Presburger formula $P_{i}$, called a node constraint, over the $m$ counters. Each edge (say, from node $i$ to node $j$ ) in $G$ is labeled with an addition vector $\vec{\Delta}_{i j}$ in $\mathrm{N}^{m}$. Essentially, $G$ defines a stateless multicounter system whose semantics is as follows. Intuitively, $G$ specifies a multicounter system with $n$ groups of counters with each group $\vec{X}_{i}$ of $m$ counters. In the system, there are no states. Each instruction is the addition vector $\vec{\Delta}_{i j}$ specified on an edge. The semantics of the instruction, when applied, is to decrement counters in group $\vec{X}_{i}$ by $\vec{\Delta}_{i j}$ and increment counters in group $\vec{X}_{j}$ by $\vec{\Delta}_{i j}$ (we emphasize the fact that each component in the vector $\vec{\Delta}_{i j}$ is nonnegative by definition). When the system runs, an instruction is nondeterministically chosen and applied. Additionally, we require that at any moment during a run, for each $i$, the constraint $P_{i}$ is true when evaluated on the counter values on group $\vec{X}_{i}$. Formally, a configuration is a tuple of $n$ vectors $\left(\vec{V}_{1}, \ldots, \vec{V}_{n}\right)$ with each $\vec{V}_{i} \in \mathrm{N}^{m}$ satisfying the node constraint $P_{i}\left(\vec{V}_{i}\right)$. Given two configurations $\mathcal{C}=\left(\vec{V}_{1}, \ldots, \vec{V}_{n}\right)$ and $\mathcal{C}^{\prime}=\left(\vec{V}_{1}^{\prime}, \ldots, \vec{V}_{n}^{\prime}\right)$, we say that $\mathcal{C}$ can reach $\mathcal{C}^{\prime}$ in a move, written $\mathcal{C} \rightarrow_{G} \mathcal{C}^{\prime}$, if there is an edge from node $i$ to node $j$ (for some $i$ and $j$ ) such that $\mathcal{C}$ and $\mathcal{C}^{\prime}$ are exactly the same except that $\vec{V}_{i}-\vec{\Delta}_{i j}=\vec{V}_{i}^{\prime}$ and $\vec{V}_{j}+\vec{\Delta}_{i j}=\vec{V}_{j}^{\prime}$. We say that $\mathcal{C}$ can reach $\mathcal{C}^{\prime}$ in $G$, written

$$
\mathcal{C} \leadsto{ }_{G} \mathcal{C}^{\prime}
$$

if, for some $t$,

$$
\mathcal{C}_{0} \rightarrow{ }_{G} \mathcal{C}_{1} \ldots \rightarrow{ }_{G} \mathcal{C}_{t}
$$

where $\mathcal{C}=\mathcal{C}_{0}, \mathcal{C}_{1}, \ldots, \mathcal{C}_{t}=\mathcal{C}^{\prime}$ are configurations. We now study the following reachability problem: given a $G$ and two Presburger formulas $P$ and $Q$, whether there are $\mathcal{C}$ and $\mathcal{C}^{\prime}$ such that $\mathcal{C} \sim_{G} \mathcal{C}^{\prime}$ and, $\mathcal{C}$ and $\mathcal{C}^{\prime}$ satisfy $P$ and $Q$, respectively.

One can show that the reachability problem is undecidable even under a special case:

Theorem 10 The reachability problem for sequential tissue-like $P$ systems $G$ is undecidable even when $G$ is a $D A G$.

We now consider the case when $G$ is atomic; i.e., each node constraint $P_{i}$ in $G$ is a conjunction of atomic linear constraints, i.e., $P_{i}$ is in the form of

$$
\bigwedge\left(\sum_{j} a_{i j} x_{i j} \# c_{i}\right)
$$

where $\# \in\{\leq, \geq\}, a_{i j}$ and $c_{i}$ are integral constants. Using a VAS to simulate an atomic sequential tissue-like $\mathrm{P}$ system, one can show: 
Theorem 11 The reachability problem for atomic sequential tissue-like P systems is decidable.

In fact, the converse of Theorem 11 can be shown, i.e., atomic sequential tissuelike $\mathrm{P}$ systems and VAS are essentially equivalent, in the following sense. Consider a VAS $M$ with $k$ counters $\left(x_{1}, \ldots, x_{k}\right)$ and a sequential tissue-like $\mathrm{P}$ system $G$ with a distinguished node on which the counters are $\left(z_{1}, \ldots, z_{l} ; x_{1}, \ldots, x_{k}\right)$. We further abuse the notation $\sim_{G}$ as follows. We say that $\left(z_{1}, \ldots, z_{l} ; x_{1}, \ldots, x_{k}\right)$ reaches $\left(z_{1}^{\prime}, \ldots, z_{l}^{\prime} ; x_{1}^{\prime}, \ldots, x_{k}^{\prime}\right)$ in $G$ if there are $\mathcal{C}$ and $\mathcal{C}^{\prime}$ such that $\mathcal{C} \sim_{G} \mathcal{C}^{\prime}$ and, $\mathcal{C}$ and $\mathcal{C}^{\prime}$, when projected on the distinguished node, are $\left(z_{1}, \ldots, z_{l} ; x_{1}, \ldots, x_{k}\right)$ and $\left(z_{1}^{\prime}, \ldots, z_{l}^{\prime} ; x_{1}^{\prime}, \ldots, x_{k}^{\prime}\right)$, respectively. We say that $M$ can be simulated by $G$ if, for all $\left(x_{1}, \ldots, x_{k}\right)$ and $\left(x_{1}^{\prime}, \ldots, x_{k}^{\prime}\right)$ in $\mathrm{N}^{k},\left(x_{1}, \ldots, x_{k}\right)$ reaches $\left(x_{1}^{\prime}, \ldots, x_{k}^{\prime}\right)$ in $M$ iff $\left(0, \ldots, 0 ; x_{1}, \ldots, x_{k}\right)$ reaches $\left(0, \ldots, 0 ; x_{1}^{\prime}, \ldots, x_{k}^{\prime}\right)$ in $G$. We say that $G$ is simple if each constraint $P_{i}$ in $G$ is a conjunction of $x_{i j} \geq c$ (open constraint) or $x_{i j} \leq c$ (closed constraint), for some constant $c$ and every $j$, where $x_{i j}$ is a counter in node $i$. Notice that if $G$ is simple then $G$ must be atomic also. One can show:

Theorem 12 Every VAS can be simulated by a sequential tissue-like $P$ system $G$ that is a DAG and simple.

For a sequential tissue-like $\mathrm{P}$ system $G$, a very special case is that there is only one counter in each node, and the instruction on an edge $(i, j)$ is $I=1$, which means that when $I$ is executed, counter $i$ is decremented by 1 , and counter $j$ is incremented by 1 . We call such a $G$ single. One can show that the reachability relation $\sim_{G}$ is Presburger definable if $G$ is a DAG and single. The proof technique is to "regulate" the reachability paths in $G$ and use reversal-bounded counter machine arguments, and then appeal to Theorem 2.

Theorem 13 The reachability relation $\sim_{G}$ is Presburger definable when sequential tissue-like $P$ system $G$ is a $D A G$ and single.

Currently, we do not know whether Theorem 13 still holds when the condition of $G$ being a DAG is removed.

As we pointed out, sequential tissue-like $\mathrm{P}$ systems are essentially stateless. To conclude this section, we give an example where some forms of sequential tissue-like $\mathrm{P}$ systems become more powerful when states are added, and hence states matter (In contrast to this, VAS and VASS (by adding states to VAS) are known to be equivalent).

Consider a sequential tissue-like P system $G$ where each node contains only one counter and, furthermore, $G$ is a DAG. From Theorem 13, its reachability relation $\sim_{G}$ is Presburger definable. We now add states to $G$ and show that the reachability relation now is not necessarily Presburger definable. $G$ with states is essentially a multicounter machine $M$ with $k$ counters $\left(x_{1}, \ldots, x_{k}\right)$ and each counter is associated with a simple constraint defined earlier. Each instruction in $M$ is in the following form:

$$
\left(s_{p}, x_{i}, x_{i+1}, s_{q}\right)
$$

where $1 \leq i<k$ and, $s_{p}$ and $s_{q}$ are the states of $M$ before and after executing the instruction. When the instruction is executed, $x_{i}$ is decremented by $1, x_{i+1}$ 
is incremented by 1 , and the simple constraint on each counter should be satisfied (before and after the execution).

Now, we show that an $M$ can be constructed to "compute" the inequality $x * y \geq$ $z$, which is not Presburger definable. We need 8 counters, $x_{1}, \ldots, x_{8}$ in $M$. The idea is that we use the initial value of $x_{3}, x_{5}$ and $x_{7}$ to represent $x, y$ and $z$, respectively, and the remaining counters are auxiliary. In particular, $x_{1}$ acts as a "warehouse" for supplying counter values. The constraint upon every counter is simply $x_{i} \geq 0$, $1 \leq i \leq 8$. Initially, the state is $s_{0}, x_{2}=0$, and all the other counters store some values. We have the following instructions:

$$
\begin{aligned}
& I_{1}=\left(s_{0}, x_{3}, x_{4}, s_{1}\right) ; \\
& I_{2}=\left(s_{1}, x_{1}, x_{2}, s_{2}\right) ; \\
& I_{3}=\left(s_{2}, x_{7}, x_{8}, s_{0}\right) ; \\
& I_{4}=\left(s_{0}, x_{5}, x_{6}, s_{3}\right) ; \\
& I_{5}=\left(s_{3}, x_{2}, x_{3}, s_{0}\right) ; \\
& I_{6}=\left(s_{0}, x_{2}, x_{3}, s_{0}\right) .
\end{aligned}
$$

Note that $s_{3}$ is the accepting state. $I_{1}, I_{2}$ and $I_{3}$ mean that, when $x_{3}$, which represents $x$, is decremented by $1, x_{2}$ will record the decrement, and $x_{7}$, which represents $z$, will also be decremented by 1 . $I_{4}$ says that during the decrement of $x_{3}, x_{5}$, which represents $y$, will be nondeterministically decremented by $1 . I_{5}$ and $I_{6}$ will restore the value of $x_{3}$, and after the restoration, the value of $x_{3}$ can never surpass the initial one (i.e., $x$ ). One can show that $x * y \geq z$ iff $M$ can reach state $s_{3}$ (the accepting state) and, at the moment, $x_{7}=0$.

\section{Conclusion}

We introduced the notion of stateless multihead two-way (respectively, one-way) NFAs and stateless multicounter systems and related them to $\mathrm{P}$ systems and vector addition systems. In particular, we investigated the decidability of the emptiness and reachability problems for these stateless automata and showed that the results are applicable to similar questions concerning certain variants of $\mathrm{P}$ systems, namely, token systems and sequential tissue-like $\mathrm{P}$ systems. Many issues (e.g., the open problems mentioned in the previous sections) remain to be investigated, and we plan to look at some of these in future work.

\section{References}

[1] Z. Dang and O. H. Ibarra. On one-membrane P systems operating in sequential mode. Int. J. Found. Comput. Sci., 16(5):867-881, 2005.

[2] S. Ginsburg and E. Spanier. Semigroups, Presburger formulas, and languages. Pacific J. of Mathematics, 16:285-296, 1966. 
[3] O. H. Ibarra. A note on semilinear sets and bounded-reversal multihead pushdown automata. Inf. Processing Letters, 3(1): 25-28, 1974.

[4] O. H. Ibarra. Reversal-bounded multicounter machines and their decision problems. Journal of the ACM, 25(1):116-133, 1978.

[5] O. H. Ibarra, Z. Dang, and Ö. Egecioglu. Catalytic P systems, semilinear sets, and vector addition systems. Theor. Comput. Sci., 312(2-3):379-399, 2004.

[6] C. Martín-Vide, Gh. Păun, J. Pazos, and A. Rodríguez-Patón. Tissue P systems. Theor. Comput. Sci., 296(2):295-326, 2003.

[7] E. Mayr. An algorithm for the general Petri net reachability problem. Proc. 13th Annual ACM Symp. on Theory of Computing, 238-246, 1981.

[8] Gh. Păun. Computing with membranes. Journal of Computer and System Sciences, 61(1):108-143, 2000.

[9] Gh. Păun. Membrane Computing, An Introduction. Springer-Verlag, 2002.

[10] L. Yang, Z. Dang, and O.H. Ibarra. Bond computing systems: A biologically inspired and high-level dynamics model for pervasive computing. Proceedings of the 6th International Conference on Unconventional Computation (UC'07), Lecture Notes in Computer Science, 2007. 